\title{
Malignant Retropharyngeal Neoplasm
}

National Cancer Institute

\section{Source}

National Cancer Institute. Malignant Retropharyngeal Neoplasm. NCI Thesaurus. Code C162828.

A primary or metastatic malignant neoplasm that affects the retropharyngeal space. 